\title{
Terapia de bacterias productoras de $\beta$-lactamasas de espectro extendido
}

\author{
RICARDO MORALES I *
}

\section{Treatment of extended spectrum betalactamases producing bacteria}

We analyze the epidemiological features related to extended spectrum B-lactamases producing Enterobacteriaceae and their therapeutic alternatives; the usefulness of cefepime, aminoglycosides, quinolones, $\beta$-lactam- $\beta$-lactamase inhibitors and carbapenems are discussed. Carbapenems represent the first choice treatment for serious infections caused by these multiresistant bacteria.

Key words: Extended specrum beta lactamases; Enteobacteriaceas; Treatment; Carbapenems; Cefepime

Concepto: Las $\beta$-lactamasas de espectro extendido (BLEE) son enzimas capaces de hidrolizar penicilinas, cefalosporinas de amplio espectro y monobactámicos que derivan de enzimas tipo TEM y SHV principalmente (descritas también de CTX, PER, OXA). Se localizan en plasmidios y son transferibles de cepa a cepa entre especies bacterianas.

La prevalencia en muchos hospitales está en aumento principalmente en Escherichia coli y Klebsiella pneumoniae.

Importancia clínica: Pacientes infectados con bacterias productoras de BLEE tienen mayor riesgo de mortalidad si son tratados con antimicrobianos a los que la bacteria tenga alto nivel de resistencia.

Otras revisiones muestran un fracaso mayor de $50 \%$ en la terapia de los pacientes con bacterias productoras de BLEE tratados con cefalosporinas, a pesar de que los tests de susceptibilidad informaban a la bacteria como susceptible.

Si se detecta la presencia de BLEE, todas las penicilinas, aztreonam y cefalosporinas incluido cefepime (no incluye cefoxitina y cefotetan, ya que característicamente las BLEE no hidrolizan cefamicinas) deben ser reportadas como resistente independientemente de la susceptibilidad informada.

Fracaso clínico: Puede originarse en la gran cantidad de bacterias en el sitio de infección, lo que se conoce como efecto inóculo. La CIM de las cefalosporinas aumenta dramáticamente cuando el inóculo aumenta de 10 a 100 veces. En algunas infecciones como endocarditis e infecciones intraabdominales, la carga bacteriana puede superar niveles de $10^{6}-10^{7} \mathrm{UFC} / \mathrm{ml}$.

\section{Epidemiología y control de infecciones}

La población más expuesta a brotes, en adultos, incluye: pacientes de UCI, pacientes con trasplantes de órganos sólidos, trasplantados de precursores hematopoyéticos, y las unidades clínicas de pacientes crónicos; en Pediatría, se concentran en UCI neonatal y pediátrica, pacientes con trasplante de órganos sólidos.

Son factores de riesgo para la adquisición de Enterobacteriaceas productoras de BLEE: enfermedades severas, hospitalización prolongada, permanencia prolongada en UCI, procedimientos invasores, presencia de catéteres intravasculares, nutrición parenteral total, ventilación mecánica, catéteres urinarios, gastrostomía, yeyunostomía o uso de sonda nasogástrica, edades extremas de la vida, hemodiálisis, úlceras de decúbito, desnutrición y bajo peso de nacimiento.

Se reconocen como reservorios y vectores: la colonización de las manos del personal de salud, termómetros, geles de ultrasonografía, catéter de oxigenación, el jabón.

\footnotetext{
*Unidad de Infectología, Servicio de Medicina Hospital Barros Luco-Trudeau

E-mail: hamburgo@vtr.net
} 
¿Cuáles son los antibióticos que seleccionan bacterias productoras de BLEE? Se describen las cefalosporinas de espectro ampliado, aztreonam, fluoroquinolonas, cotrimoxazol, aminoglucósidos y metronidazol.

Para el control de infecciones causadas por cepas con BLEE, deben ser enfatizadas para evitar su diseminación, el lavado de manos, el aislamiento de contacto, la decontaminación habitual del ambiente, el uso de instrumental exclusivo, la individualización de los pacientes, políticas de reingreso de pacientes, desarrollar y controlar el cumplimiento de protocolos de uso de antimicrobianos, efectuar focus en cefalosporinas de amplio espectro, principal antibacteriano a controlar en presencia de brotes (policlonales), y finalmente el oportuno switch de terapia empírica para infecciones severas, a otra clase de antimicrobianos.

\section{Terapia antimicrobiana contra las BLEE: Alternativas}

- Inhibidores de $\beta$-lactamasas: Existen recomendaciones diversas en cuanto al reporte de susceptibilidad a piperacilina/tazobactam ampicilina/sulbactam y amoxicilina/ácido clavulánico. Hay comunicaciones de tratamiento exitoso de infecciones causadas por bacterias productoras de BLEE pero la susceptibilidad in vivo puede ser enzima-específica. Así, BLEE derivadas de TEM son más susceptibles a piperacilina/tazobactam que las derivadas de SHV. Su actividad esta influenciada por el efecto inoculo, regímenes de administración del fármaco y el aumento de cepas con mutaciones en porinas.

Existe una limitada experiencia clínica para tratamiento de infecciones serias. Estos agentes podrían ser considerados como alternativa terapéutica.

- Quinolonas: Alternativas atractiva de tratamiento pero ya se reporta un aumento de asociación entre BLEE y resistencia a quinolonas, que es usualmente cromosomal. Han sido documentada la resistencia plasmidial con $\beta$-lactamasas tipo Amp C, sumado a una alteración en porinas.

- Aminoglucósidos: Merece consideraciones similares a quinolonas.

Las BLEE no tienen efecto intrínseco en su actividad, pero la resistencia a aminoglucósidos puede co-transferirse con BLEE a través de plasmidios. Los aminoglucósidos no son una alternativa terapéutica apropiada como monoterapia.

- Cefamicinas: Cefamicinas (cefoxitina y cefotetan) son estructuralmente más estables a la hidrólisis por BLEE, muchas bacterias son susceptibles in vitro. La información de tratamiento de infecciones graves con cefamicinas es muy limitada y hay reportes de fracaso clínico debidos a la emergencia de resistencia intratratamiento por el desarrollo de mutación en porinas.

Se ha observado un aumento de cepas que expresan múltiples $\beta$-lactamasas Amp C que originan resistencia a este grupo.

- Oximino $\beta$-lactámicos: El dilema de cefepime. Cefepime es activo contra muchas cepas productoras de BLEE, particularmente de enzimas derivadas de SHV. Sin embargo, la susceptibilidad disminuye con el aumento del inoculo en test de susceptibilidad in vitro $\mathrm{y}$ en modelos in vivo. Este es el llamado efecto inoculo: aumento en 8 veces la CIM con mayor inóculo bacteriano; ha sido observado en cefepime y cefotaxima, es menos importante en carbapenems, y de trascendencia intermedia en piperacilina/tazobactam.

Existe un reporte nacional de 75 cepas de $K$. pneumoniae productoras de BLEE 2,6\% resistente, y 12 cepas de E. coli $25 \%$ resistente.

El resultado clínico es bueno cuando el sitio de infección es urinario (alcanza mayores concentraciones locales) ¿Está indicado en infecciones severas?

Mantiene mejor actividad que piperacilina/ tazobactam (en un análisis farmococinéticofarmacodinámico). Se ha conocido de fallas clínicas en reportes bacteriológicos que lo informaban como sensible (bacteriemia por K. pneumoniae).

No debe indicarse como primera línea en infecciones graves por bacterias productoras de BLEE, y si se usa, la dosis debe ser mayor o igual a 2 grs cada $12 \mathrm{hrs}$ y en combinación con otro agente activo (aminoglucósidos o fluoroquinolonas).

- Carbapenems: Representan la terapia de elección en bacterias productoras de BLEE (imipenem-meropenem). Son resistentes a la hidrólisis y tienen excelente actividad in vitro. Diversos estudios clínicos avalan su uso; Meyer et al exhiben una mejor sobrevida de 
los pacientes tratados con imipenem durante un brote de $K$. pneumoniae productora de BLEE. Se ha descrito mecanismos de resistencia por carbapenemasas mediadas por plasmidios, metalo $\beta$-lactamasas y proteasas de espectro extendido; afortunadamente son infrecuentes. Resistencia debido a alteraciones en las porinas ha sido observada en cepas de K. pneumoniae. Debe tenerse especial precaución en la prescripción de carbapenems por la aparición de Acinetobacter spp resistente, Stenotrophomonas maltophilia o Pseudomonas spp.

- Nuevas alternativas terapéuticas: Ertapenem y faropenem tienen excelente actividad contra bacterias productoras de BLEE ( $K$. pneumoniae, E. coli, etc) y cefalosporinasas Amp C. Tienen la ventaja farmacocinética de una vida media larga que permite indicar una dosis diaria.

\section{Conclusiones}

La habilidad para tratar exitosamente infecciones por microorganismos resistentes requiere de un enfoque multifactorial combinado con investigación continua y el desarrollo de nuevos antibacterianos, el uso prudente de los antimicrobianos existentes, manteniendo el énfasis en las medidas de control de infecciones.

\section{Resumen}

Se analizan aspectos epidemiológicos de Enterobacteriaceas productoras de b-lactamasas de espectro expandido y alternativas terapéuticas; dentro de éstas se discute el rol de cefepime, aminoglucósidos, quinolonas, b lactámicos combinados con inhibidores de b -lactamasas y carbapenems. Estos últimos constituyen la elección en la terapia de infecciones graves causadas por estas bacterias multiresistentes.

\section{Bibliografía}

1.- Rupp M E, Fey P D. Extended spectrum $\beta$-lactamase (ESBL)-producing Enterobacteriaceae: considerations for diagnosis, prevention and drug treatment. Drugs 2003; 63(4): 353-65.

2.- Paterson D L, Ko W C, Von Gottberg A et al. Outcome of cephalosporin treatment for serious infections due to apparent susceptible organisms producing extended-spectrum beta-lactamases: implications for the clinical microbiology laboratory. J Clin Microb 2001; 39(6): 2206-12. Review.

3.- National Committee for Clinical Laboratory Standards. 2003. Methods for dilution antimicrobial susceptibility test for bacteria that grow aerobically ; Approved Standard- Sixth Edition. M7A6, Vol 23 (2): 12.

4.- Medeiros A A, Crellin J. Comparative susceptibility of clinical isolates producing extended spectrum betalactamases to ceftibuten: effect of large inocula. Pediatr Infect Dis J 1997; 16(3 Suppl): S49-55.

5.- Rahal J J, Urban C, Horn D et al. Class restriction of cephalosporin use to control total cephalosporin resistance in nosocomial Klebsiella. JAMA 1998 Oct 14; 280(14): 1233-7.

6.- Pirota L, Aubé H, Doise J M, Vincent-Marti M. Spreed of extended-epectrum beta-lactamase-producing Klebsiella pneumoniae: Are Beta-Lactamase Inhibitors of therapeutic value?. Clin Infect Dis 1998; 27: 7680.

7.- Pena C, Pujol M, Ardanuy $\mathrm{C}$ et al. Epidemiology and successful control of a large outbreak due to Klebsiella pneumoniae producing extended-spectrum betalactamases. Antimicrob Agents Chemother 1998; 42(1): 53-8.

8.- Thomson K S, Moland E S. Cefepime, piperacillintazobactam, and the inoculum effect in tests with extended-spectrum beta-lactamase-producing Enterobacteriaceae. Antimicrob Agents Chemother 2001 Dec; 45(12): 3548-54.

9.- Bradford P A. Extended-spectrum beta-lactamases in the 21th century: characterization, epidemiology, and detection of this resistance threat. Clin Microbiol Rev 2001; 14(4): 933-51.

10.- Patterson J E, Hardin T C, Kelly C A, García R C, Jorgensen $\mathrm{J}$ H. Association of antibiotic utilization measures and control of multiple-drug resistance in Klebsiella pneumoniae. Infect Control Hosp Epidemiol 2000; 21(7): 455-8.

11.- Spanu T, Luzzaro F, Perilli M, Amicosante G, Toniolo A, Fadda G; Italian ESBL Study Group. Occurrence of extended-spectrum beta-lactamases in members of the family Enterbacteriaceae in Italy: implications for resístance to beta-lactams and other antimicrobial drugs. Antimicrob Agents Chemother 2002; 46(1): 196-202.

12.- Paterson D L, Mulazimoglu L, Casellas J M et al. Epidemiology of ciprofloxacin resistance and its relationship to extended-spectrum beta-lactamase production in Klebsiella pneumoniae isolates causing bacteremia. Clin Infect Dis 2000; 30(3): 473-8.

13.- Martínez-Martínez L, Hernández-Alles S, Alberti S, Tomas JM, Benedi VJ, Jacoby GA. In vivo selection of porin-deficient mutants of Klebsiella pneumoniae with increased resistance to cefoxitin and expandedspectrum-cephalosporins. Antimicrob Agents Chemother 1996; 40(2): 342-8.

14.- Pangon B, Bizet C, Bure A, Pichon F, Philippon A, Regnier B, and Gutmann L. In vivo selection of cephamycin-resistant, porin-deficient mutant of Klebsiella pneumoniae producing a TEM-3 betalactamase. J Infect Dis 1989; 159: 1005-6.

15.- Bradford P A, Urban C, Mariano N, Projan S J, Rahal $\mathrm{J} \mathrm{J}$; Bush K. Imipenem resistance in Klebsiella pneumoniae is associated with the combination of ACT-1, a plasmid-mediated AmpC beta-lactamase, and the foss of an outer membrane protein. Antimicrob Agents Chemother 1997 Mar; 41(3): 563-9

16.- Ambrose P G, Bhavnani S M, Jones R N. Pharmacokineticspharmacodynamics of cefepime and piperacillin-tazobactam against Escherichia coli and Klebsiella pneumoniae strains producing extended-spectrum beta-lactamases: report from the ARREST program. Antimicrob Agents Chemother 2003; 47(5): 1643-6. 
17.- Granier S, Nicolas-Chanoine M, Nguyen Van J C, Leflon-Guibout V, Kitzis M, Goldstein F. False susceptibility of Klebsiella oxytoca to extended spectrum cephalosporins. Program and Abstracts of the $41^{\text {th }}$ ICAAC Chicago, December 16-19, 2001 Abstract K 1180.

18.- Meyer K S, Urban C, Eagan J A, Berger B J, Rahal J J. Nosocomial outbreak of Klebsiella infection resistant to late-generation cephalosporins. Ann Intern Med 1993 1; 119(5): 353-8.

19.- Paterson D L, Ko W C, Mohapatra S, Van Gottberg A, et al. Klebsiella pneumoniae bacteremia: impact of extended-spectrum beta-lactamase (ESBL) production in a global study of 216 patients. Program and Abstracts of the $37^{\text {th }}$ ICAAC 1997, Toronto Sept 28-Oct 1 Abstract J-210.

20.- Wong-Beringer A. Therapeutic challenges associated with extended-spectrum, $\beta$-lactamase-producing Escherichia coli and Klebsiella pneumoniae Pharmacother 2001; 21: 583-92.

21.- Mell S, Zemelman C, Bello H, Domínguez M, González G, Zemelman R. Propiedades microbiológicas, clasificación y relación estructura-actividad de cefalosporinas e importancia de las cefalosporinas de cuarta generación. Rev Chil Infect 2001; 18(1): 7-19.

22.- Cunha B. Ertapenem: a review of its microbiologic, pharmacokinetic and clinical aspects. Drugs Today 2002, 38(3): 195-213. 